\title{
Contour Extraction of Skin Tumors Using Visual Attention and GVF-Snake Model
}

\author{
Li Ma, Tianzhen Su \\ ${ }^{1}$ College of Life Information \& Instrument Engineering, Hangzhou Dianzi University, Hangzhou, China \\ ${ }^{2}$ School of Automation, Hangzhou Dianzi University, Hangzhou, China \\ Email: mali@hdu.edu.cn, 225zhisuzi@sina.com.cn
}

Received 2013

\begin{abstract}
Contour extraction of skin tumors accurately is an important task for further feature generation of their borders and surfaces to early diagnose melanomas. An integrated approach, combining visual attention model and GVF-snake, is proposed in the paper to provide a general framework for locating tumor boundaries in case of noise and boundaries with large concavity. For any skin image, the visual attention model is implemented to locate the Region of Interests (ROIs) based on saliency maps. Then an algorithm called GVF-snake is utilized to iteratively drive an initial contour, deriving from the extracted ROIs, towards real boundary of skin tumors by minimizing an energy function. It is shown from experiments that the proposed approach exceeds in two aspects compared with other contour-deforming methods: 1) initial contours generated from saliency maps are definitely located at neighboring regions of real boundaries of skin tumors to speed up converges of contour deformation and achieve higher accuracy; 2) the method is not sensitive to noises on skins and initial contours extracted.
\end{abstract}

Keywords: Visual Attention; GVF-Snake; Contour Extraction; Skin Tumors

\section{Introduction}

Melanoma is known as one of the most malignant skin tumors probably appearing on any parts of human bodies. Clinical studies show that early diagnosis can effectively improve the cure rate. In CAD systems, identifying and extracting skin tumors accurately is the important stage as the further $\mathrm{ABCD}$ diagnosing rules [1], $\mathrm{A}$ (asymmetry), $\mathrm{B}$ (border irregularity), C (color) and D (diameter), are highly depended on the extracted tumor contours or ROIs.

Extractions of skin contours, especially for melanomas, are considered as a challenging task as they appearing variety in color surfaces and geometrical irregularity along the tumor contours. There are many research works in literatures on contour extractions for skin tumors. Among them, a popular approach is the active contour models called Snakes [2] based on contour evolutions towards the optimal positions by minimizing its energy. The disadvantages of the Snakes algorithm are: 1) the initial contour should be provided manually; 2) deformable contours might not reach the object boundaries in case of weaker gradients in images. To overcome the influences of weaker gradients, an algorithm of GVFsnake [3] is presented by introducing a novel external

*This work is supported by National Scientific Foundation of China (60775016). force, Gradient Vector Flow, to generate a distributed force field driven deformed contour towards object boundaries with large concavity. Unfortunately the initial curve is still needed by users for either GVF-snake or ASM [4] and converges of deformed contours are sensitive to initial curves. There are some modified versions on initial contour generation but no general framework for reliable formation of initial contours [5,6].

In recent years, visual attention model becomes a hot research field in video monitoring systems to identify interested objects from visual information of environments. It is a process of selecting information based on saliency in images (bottom-up) and on task-related prior knowledge (up-down). The advantage of the model is that it could drive attention to focus on candidate objects even only in bottom-up module (data-driven). The motivation of the paper is to build a general model for automatically extracting contours of skin tumors with robustness and accuracy. In this paper an integrated approach of visual attention model and GVF-snake are proposed to perform automatic boundary extraction of skin tumors. The proposed algorithm is illustrated in Figure 1 where dashed block indicates sub-task of the system. It is shown in Figure $\mathbf{1}$ that there are three subblocks in the proposed algorithm for the contour extraction. The first one refers to visual attention based ROI 


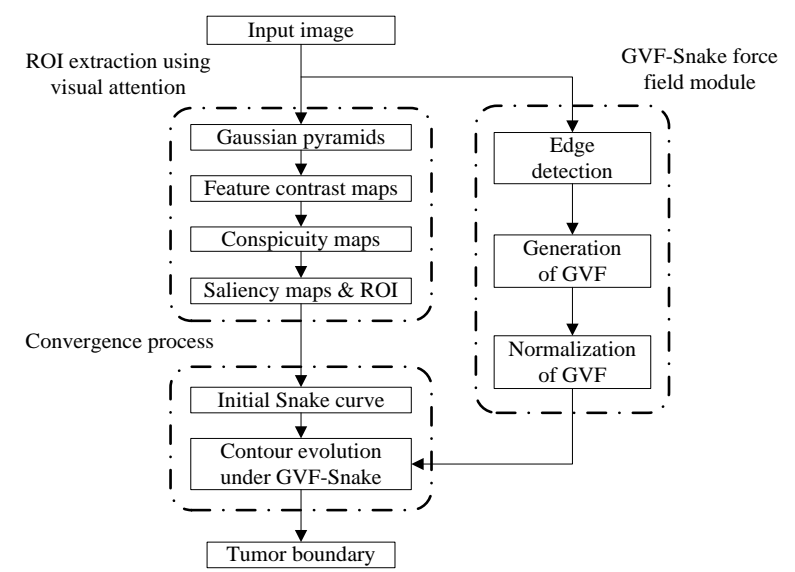

Figure 1. The proposed method for contour extraction of skin tumors.

extraction, and then GVF field is calculated to present driven force map of the input image. Finally initial contour from the extracted ROI is iteratively evolved using GVF-snake model to locate the actual boundaries of skin tumors based on the criteria of energy minimization.

\section{Bottom-Up Saliency Map Formulation}

\subsection{Visual Attention Mechanism}

The model of bottom-up salient region selection presented by Itti-Koch is based on center-surround mechanism in bottom-up way [7]. Firstly, the input image is processed progressively by Gaussian low-pass filter and then sub-sampled to generate the pyramids of multi-feature maps with nine spatial scales, where Layer 0 is an original image and the length and width in next layer is reduced by half than the previous layer. Each feature, such as intensity, color and orientation in general, is then computed by a set of linear "center-surround" operations akin to visual receptive fields. Center-surround is implemented in the model as the difference between fine and coarse scales: The center is a pixel at scale

$c=\{2,3,4\}$, and the surround is the corresponding pixel at scale $s=c+d$, with $d \in\{3,4\}$. So the feature contrast map, the region should be paid attention to, is generated by interpolation to the finer scale and point-bypoint subtraction between center and surrounding layers.

\subsection{Saliency Maps and ROI Generation}

For images of skin tumors, color and intensity are key visual features in human vision while orientations are less-relevant to identifications of skin tumors. In particular, 6 for intensity features and 12 for color features from both RG and BY channel of color differences [7]. After the 18 feature contrast maps are formed, the normalization is taken for each feature contrast maps. Then feature maps in either color and intensity sub-group are combined into two conspicuity maps in scale four: $\bar{I}$ for intensity (Equation (1)) and $\bar{C}$ for color (Equation (2)).

$$
\begin{aligned}
& \bar{I}=\stackrel{4}{\oplus} \underset{c=2}{\stackrel{c+4}{\oplus}} \mathrm{N}(I(c, s)) \\
& \bar{C}=\bigoplus_{c=2}^{4} \stackrel{c+3}{\oplus}\left[\begin{array}{c}
c=c+3 \\
s
\end{array}[\mathrm{~N}(R G(c, s))+\mathrm{N}(B Y(c, s))]\right.
\end{aligned}
$$

where “ $\oplus$ ” is defined as across-scale addition. It is implemented by reduction of each map to scale four and point-by-point addition.

As for the final saliency map $S$, it is ineffective using conventional method based on the linear combination of the two conspicuity maps as malignant tumors with rough surfaces in intensity space and variety in color space are quite different from common objects with even surfaces. So a nonlinear operator is proposed in the paper for the final fusion of saliency map for objects with uneven surfaces. The saliency value at each pixel $(x, y)$ is computed as:

$$
S(x, y)=\min \mathrm{N}(\bar{I}(x, y)), \mathrm{N}(\bar{C}(x, y)),)
$$

The generation of saliency map based on visual attention is presented in Figure 2, where Figure 2(a). is an original image of a melanoma with uneven color distributions and irregular boundary. The conspicuity maps, darker region referring to higher attention area, for both intensity and color are represented in Figure 2(b) and Figure 2(c) respectively. Figure 2(d) is the final saliency map using Equation (3). It is noted from the figure that color saliency map works better than intensity one.

At last, ROIs would be further extracted from saliency map. The procedure of ROI extraction is described as following:

1) Threshold the saliency map to get a binary image.

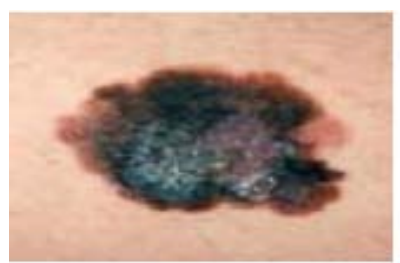

(a)

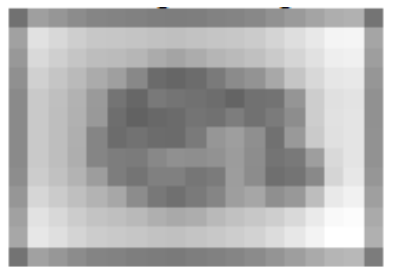

(c)

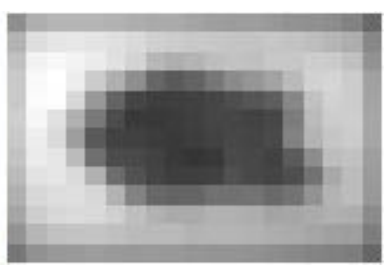

(b)

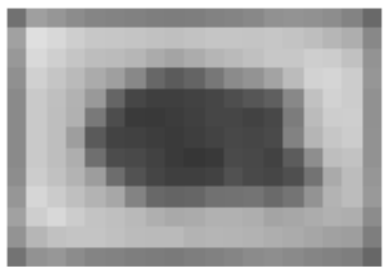

(d)
Figure 2. Formation of the saliency map of a melanoma. (a) Original image; (b) Color channel; (c) Intensity channel; (d) The saliency map. 
2) Take the opening operation to remove some noises.

3) If there are several ROIs, then the ROI candidates are marked and the one with the largest area is chosen to be the final ROI.

\section{Contour Evolution by GVF-Snake}

Snakes [2], also active contours, firstly proposed by Kass are deformable curves within an image domain that can move under the influences of internal forces, coming from and within the curve itself, and external forces computed from the image data. The curve evolution under both internal and external forces is mathematically described as a process of energy minimization in Equation (4).

$$
\begin{aligned}
E_{\text {snake }} & =\int_{0}^{1}\left(E_{\text {int }}(v(s))+E_{\text {ext }}(v(s))\right) d \\
& =E_{\text {int }}+E_{\text {ext }}
\end{aligned}
$$

where $E_{\text {snake }}$ refers to a deformable curve energy, $E_{\text {int }}$ represents internal forces and $E_{e x t}$ corresponds to external forces.

\subsection{Gradient Vector Flow Snake}

In the traditional Snakes, $E_{e x t}$ is computed by the negative gradient of the input image $\left(E_{\text {ext }}=-|\nabla I(x, y)|^{2}\right)$.

Although it is simple and easy to be implemented, there are two key drawbacks both on setting an initial curve close to the true boundary and hardly converging to boundary concavity. In order to solve these difficulties, GVF [8] method is proposed by Xu Chenyang and Prince in 1997. In this model, a new version of external forces called gradient vector flow (GVF) fields is presented. These fields are dense vector fields derived from images by solving a pair of decoupled linear partial differential equations which diffuses the gradient vectors of a binary edge map of the image to minimize an energy function.

1) GVF formation. For any gray-scale image $I(x, y)$, its GVF is defined as $V(x, y)=[u(x, y), v(x, y)]$ where $(x, y)$ represents the coordinate. Then an energy function $\varepsilon$ is defined from the GVF field and the binary edge map $\nabla f(x, y)$ of $I(x, y)$ :

$$
\varepsilon=\iint \mu\left(u_{x}{ }^{2}+u_{y}{ }^{2}+v_{x}{ }^{2}+v_{y}{ }^{2}\right)+|\nabla f|^{2}|\vec{V}-\nabla f|^{2} d x d y
$$

where $\mu$ is a regularization parameter governing the tradeoff between the first and the second term.

GVF field could be calculated by solving the Euler equations of Equation (5) to minimize the energy function.

$$
\left\{\begin{array}{l}
u=u+\mu \nabla^{2} u-\left(u-f_{x}\right)\left(f_{x}{ }^{2}+f_{y}{ }^{2}\right) \\
v=v+\mu \nabla^{2} v-\left(v-f_{y}\right)\left(f_{x}{ }^{2}+f_{y}{ }^{2}\right)
\end{array}\right.
$$

where $\nabla^{2} u=\frac{d^{2} u}{d_{x}{ }^{2}}+\frac{d^{2} u}{d_{y}{ }^{2}}$ and $\nabla^{2}$ is the Laplacian Operator, $\mu$ should be set according to the amount of noise present in the image (more noise, higher $\mu$ ).

2) GVF-snake. The active contour that uses the GVF field as its external force is named as a GVF-Snake. It effectively expands the scope and intensity of the external force. As for GVF-snake considered, external force term $-\nabla E_{e x t}$ in the energy functional $E_{t}(s, t)$ of Snake [2] (Equation (7)) is replaced with $V(x, y)$ :

$$
E_{t}(s, t)=\alpha x^{\prime \prime}(s, t)-\beta x^{\prime \prime \prime}(s, t)-\nabla E_{\text {ext }}
$$

where $x=\left[x_{i}, y_{i}\right]$ is the $i$ th pixel on the current contour. The contour evolution is iteratively performed using Equation (8) by minimizing the Equation (7).

$$
\left\{\begin{array}{l}
x_{t}=(A+\gamma I)^{-1}\left(\gamma x_{t-1}-u\left(x_{t-1}, y_{t-1}\right)\right) \\
y_{t}=(A+\gamma I)^{-1}\left(\gamma y_{t-1}-v\left(x_{t-1}, y_{t-1}\right)\right)
\end{array}\right.
$$

where, the matrix $A+\gamma I$ is a pentadiagonal banded matrix related to parameter $\alpha, \beta$ in Equation (7). As mentioned above, the curve profile could move into the concave boundary regions because there are stronger external forces but still needs setting initial curves.

\subsection{Contour Extraction of Skin Tumors Based on Visual Attention and GVF-Snake}

As mentioned above, GVF-snake provides the solution for one of major defects in conventional snake with respect to contour concavity, but its initial contour is still given by users. In this way the final curves may not be guaranteed to reach the actual boundaries of objects especially when initial contours are located at the weaker regions of GVF fields. However, visual attention based ROI extraction would make contribution to initial contour generation automatically and ensure the contour derived from saliency map to locate at neighboring regions of real object boundary. The proposed algorithm of combination of visual attention and GVF-Snake for skin contour extractions is described below:

Step 1. Visual attention is used for the input image to obtain the ROI of a skin tumor from a saliency map (see the Section 2).

Step 2. The GVF field of input image is generated using the Equation (6) where edge gradient $\nabla f(x, y)=\left[f_{x}, f_{y}\right]$ can be calculated from the gray scale image $I(x, y)$.

Step 3. The initial contour $s=\left(x_{1}, x_{2}, \cdots x_{n}\right)$ is extracted from the contour of ROI obtained in step1.

Step 4. The initial contour is iteratively evolved based on Equation (8) which moves the deformable contour towards the real contour of skin tumor in the image. Iteration isn't stopped until the most of points on the contour are not change. Then the final result of contour ex- 
traction is obtained.

\section{Experimental Results}

To verify the effectiveness of our proposed method for skin contour extraction, sample images were all downloaded from the Dermnet Skin Disease Image Atlas database at website http://www.dermnet.com. Each image was of size $300 \times 200$ pixels and working platform is matlab 7.11 on Windows 7. The proposed method in this paper is compared with other approaches (GVF-snake and ASM).

\subsection{Experiments of Contour Extraction for Skin Tumors}

Experiments are taken on three aspects: sensitivity on initial contour, robustness from noises and accuracy measure. Only melanomas are chosen from the database as accurate extraction of their boundaries is more difficult than moles.

In Figure 3 comparison among several methods is presented for a given image of a melanoma where curve marked with dashed thin line (" $x \cdots x$ ") in white indicates the initial contour and the one marked with "_-" in red represents the final boundary of the skin tumor. The contour extracted on the proposed algorithm is given in Figure 3(a) where the initial curve generated from visual attention is located in the internal region of the tumor and the final contour reaches the real boundary of the tumor. The results of other methods, ASM and GVF-snake, are shown in Figure 3(b), Figure 3(c) and 3(d) respectively. As for ASM method, the initial contour in Figure 3(b) derived from the averaged boundary in database of skin tumors and it is noted that the final contour fails to reach the real boundary as the range of contour deforming is limited by its model parameters. The GVF-snake is

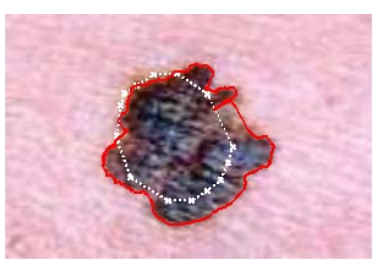

(a)

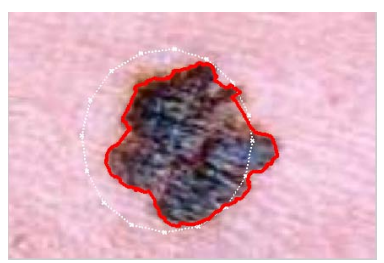

(c)

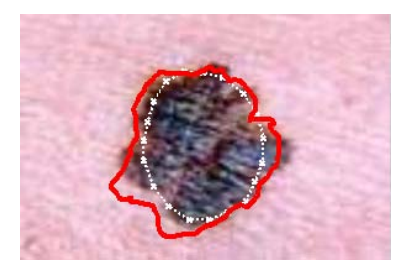

(b)

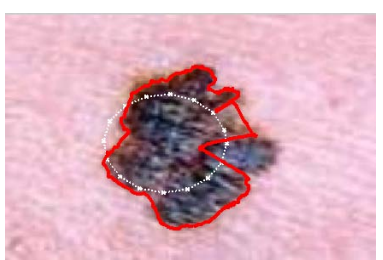

(d)
Figure 3. Comparison results of contour extraction on differenrt mothods, (a) proposed method; (b) ASM algorithm; (c) GVF-snake; (d) GVF-snake. sensitive to the initial contour setting when given two different initial contours (see Figures 3(c) and (d)).

The experiment in Figure $\mathbf{4}$ is for testing and comparing methods above when noise exists. In Figure 4, there are some hairs around a skin tumor. It is shown that the final converged contour (Figure 4(a)) is located at actual positions of the tumor boundary using proposed method while either ASM (Figure 4(b)) method or GVF-snake (Figures 4(c) and (d)) have poor results. It is the hairs (noise) that interfere with moving direction of ASM and GVF field for contour deformations. It is known from experiments that the proposed approach (visual attention \& GVF-snake) performs better than the other two. It is ensured that the initial contours from visual attention model are located near boundaries of skin tumors as the region of boundaries with strong feature contrast would have larger values of saliency.

\subsection{Accuracy Measurement}

Accuracy of contour extractions is an important index for algorithm evaluation. Apart from conventional accuracy measure, another accuracy measure is given in the paper using binary logics.

$$
m_{a}=1-\frac{A\left(M_{R} \oplus M_{F}\right)}{A\left(M_{R}\right)} \times 100 \%
$$

where $m_{a}$ is the accuracy index, $A(\cdot)$ means calculating the area of a region. $M_{R}$ is the region surrounded by an actual contour marked by users and $M_{F}$ represents the region surrounded by the final contour extracted. $\oplus$ complete the operation of XOR.

The experimental results are given in Table $\mathbf{1}$ where accuracy and executing time are compared. Ten sample images are selected randomly from sub-database of melanomas. There are three methods, VA\& GVF-snake, GVF-snake and ASM used in the experiments. For the

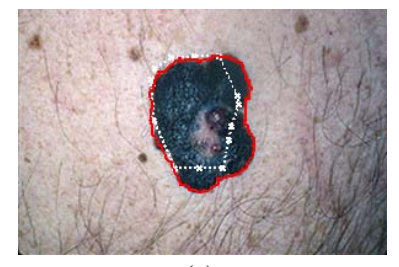

(a)

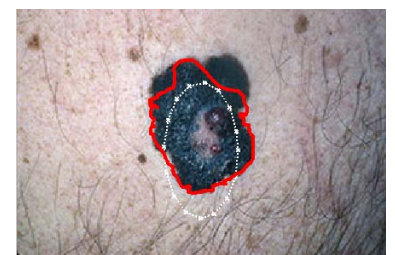

(c)

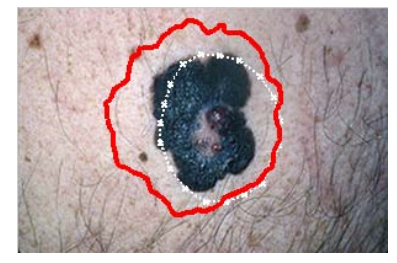

(b)

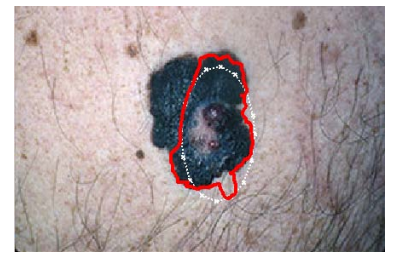

(d)
Figure 4. Comparison results for contour extraction on different mothods. (a) Proposed method; (b) ASM algorithm; (c) GVF-snake; (d) GVF-snake. 
Table 1. Performance comparisons.

\begin{tabular}{cccc}
\hline Method & VA \& GVF-Snake & GVF-Snake & ASM \\
\hline Accuracy & & & \\
Minerage & $91.145 \%$ & $74.322 \%$ & $55.441 \%$ \\
Minimum & $86.197 \%$ & $20.615 \%$ & $36.35 \%$ \\
Executing time & $3.523 \mathrm{~s}$ & $4.095 \mathrm{~s}$ & $34.472 \mathrm{~s}$ \\
\hline
\end{tabular}

accuracy measure in Equation(9), two iterms, both averaged value of $m_{a}$ and its minimum in the testing group are presented.

It is shown in Table 1 that: 1) the best performances on extraction accuracy are achieved by the proposed method in this paper; 2) the executing time of the VA \& GVF-Snake is the least among the three methods. And it is worst for ASM on both accuracy and executing time.

\section{Conclusion}

Model based contour extraction of skin tumor faces difficulties in initial contour setting as it may be located at weaker gradient fields and failed to reach real boundary of a tumor. The proposed method combines visual attention model with GVF-snake to provide a solution for the problems above. The visual attention, using feature contrast based saliency maps, generate an initial contour near the ideal boundary. Then it is further moved by GVFsnake to secure converges to real border of a tumor. It is indicated from the experiments that the proposed method in this paper has three advantages over ASM model and GVF-snake (both are sensitive to initial contours): 1) initial contours are guaranteed to be near real boundaries to make the process of curve evolution faster with higher accuracy; 2) experimental results are not sensitive to noises; 3) Initial contour is generated automatically. The method is suitable for contour extractions of objects which have large concavity or noises exist in the background of images.

\section{References}

[1] I. Maglogiannis, S. Pavlopoulos and D. Koutsouris, “An Integrated Computer Supported Acquisition, Handling and Characterization System for Pigmented Skin Lesions in Dermatological Images," Information Technology in Biomedicine, Vol. 9, No. 1, 2005, pp. 86-98.

[2] M. Kass, A. Witkin and D. Terzopoulos, "Snakes: Active Contour Models," International Journal of Computer Vision, Vol. 1, 1998, pp. 321-331. http://dx.doi.org/10.1007/BF00133570

[3] C. Xu and J. L. Prince, "Snakes, Shapes and Gradient Vector Flow," IEEE Transactions on Image Processing, Vol. 7, 1998, pp. 359-369. http://dx.doi.org/10.1109/83.661186

[4] T. Cootes, "An Introduction to Active Shape Models," Image Processing and Analysis, 2000, pp. 223-248.

[5] C.-C. Liu, C.-Y. Tsai, T.-S. Tsui and S.-S. Yu, “An Improved GVF Snake Based Breast Region Extrapolation Scheme for Digital Mammograms,” Expert Systems with Applications, 2011, pp. 1-6.

[6] B. R. Wu, M. Xie, G. Li and J. J. Gao, "Medical Image Segmentation Based on GVF Snake Model," IEEE Conference on Second International Intelligent Computation Technology and Automation, Vol. 1, 2009, pp. 637-640.

[7] L. Itti, C. Koch and E. Niebur, "A Model for SaliencyBased Visual Attention for Rapid Scene Analysis,” IEEE Transactions on Pattern Analysis and Machine Intelligence, Vol. 20, No. 11, 1998, pp. 1254-1259.

[8] J. L. Prince and C. Xu, “A New External Force Model for Snakes," Proceedings of the IEEE Computer Society Conference on Computer Vision and Pattern Recognition, 1997, pp. 66-71. 